\title{
ORIGINAL
}

Yasser M. Shabana (iD - Mohamed A. Samy •

Mohamed A. Abdel-Aziz • Mohamed E. Hindawi •

Mohamed G. Mosry • Abdul-Rahman M. Albarawy •

Mazen M. Omar • Ayman A. Mohamed • Ahmed A. Attia

\section{Enhancing the performance of micro-biosensors by functionally graded geometrical and material parameters}

Received: 26 August 2020 / Accepted: 29 January 2021 / Published online: 20 February 2021

(C) The Author(s), under exclusive licence to Springer-Verlag GmbH, DE part of Springer Nature 2021

\begin{abstract}
Most recently, the whole world is struggling against the virulent pandemic COVID-19. Due to the unbounded global spread of the disease, having biosensors with high performance such as high sensitivity and accuracy is of utmost importance. In this paper, the effects of various parameters on the behaviors of microbiosensors are investigated in order to enhance their performance. These parameters are related to the geometry and material, and they are assumed to be gradually changing in the longitudinal direction of the biosensor according to a power law. Therefore, they are called functionally graded geometrical and material parameters. Another aspect is when considering microcantilever-based biosensors, the main behavior parameter is the deflection at the free end. In the analyses, the influences of the surface stress and van der Waals intermolecular forces are taken into account. Also, the total energy of the beam, which is the combination of the van der Waals energy and the elastic strain energy, is accomplished. In addition, the equivalent force causing the deflection is also evaluated using Castigliano method for two cases. These cases account for a concentrated force at the free end and a distributed load along the biosensor, respectively. Since the governing equations account for the size dependency and the considered parameters are functions of the position, the solution is too complex to be achieved analytically, and therefore, numerical methods are applied. For uniform biosensors made of homogeneous materials, or in other words when all parameters are not varying with the position, the obtained results are compared with those in the literature, and good agreement is obtained. On the other hand, the performance, which include sensitivity and limit of detection, of functionally graded biosensors can be enhanced by proper choices of the considered parameters and the corresponding exponent of the gradation function.
\end{abstract}

Keywords Performance - Biosensor - Functionally graded parameters · Van der Waals energy · Deflection . Force

\section{Introduction}

More than a 100 years ago, Stoney [1] observed in his experiment that by depositing a thin metal film on a thick substrate, the system would be in a state of tension or compression due to the induced residual stresses. He derived his formula, which relates the curvature of two-layer beam and the induced surface stress, that became the basis in measuring surface stresses in microcantilever structures. The surface stress acting on an isotropic material tends to increase or decrease the surface, and therefore tensile or compressive stress is induced. If this stress is not compensated by an equal one on the opposite side, the beam will bend [2]. Based on this observation, microcantilever can transduce a chemical signal into mechanical motion with high sensitivity [2].

Y. M. Shabana $(\varangle)$ · M. A. Samy · M. A. Abdel-Aziz · M. E. Hindawi · M. G. Mosry · A.-R. M. Albarawy · M. M. Omar · A. A. Mohamed · A. A. Attia

Faculty of Engineering, Helwan University, P.O. Box 11718, Mataria, Cairo, Egypt

e-mail: Yasser.shabana@gmail.com 
The origins of surface stresses that cause the deflection are also investigated [3]. These sources are mainly attributed to the intermolecular forces, hydrophobic forces, steric interaction, entropic forces, and van der Waal interactions. The dominant source that causes the deflection depends on the type of biochemical reaction that occurs on the cantilever surface.

Lim et al. [4] derived a theoretical model to investigate the cantilever deflection under the effect of van der Waal forces. The implemented model was divided into two models: the continuum model, which deals with an energy approach, and the atomistic model, which considers the effect of van der Waal forces resulted from adsorbed molecules on the surface. Also, Dareing et al. [5] developed a model where they employed adsorption-induced surface stress for microcantilever beams based on the molecular interaction between the biomolecules and the surface of the cantilever. The model was concerned with the intermolecular interaction forces between the adsorbed atoms and the substrate layer. They compared their model to experimental results tested on adsorption of mercury atoms on a cantilever with a thin layer of gold. In addition, Zhang et al. [6] presented an energy method, which is based on interatomic interactions, to predict the deflection of a uniform cantilever induced by adsorbed atoms/molecules with certain density. The surface effects were incorporated as the cantilever was modeled as a sandwich beam containing a bulk layer, which made of homogenous and uniform material, between two surface layers. They demonstrated that physisorption can induce distinctly different deformation behaviors of cantilevers, which depend on both the adatoms and the material property of the substrate.

Functionally graded materials (FGMs) are inhomogeneous composites, with continuous compositional variation from ceramic to metal [7]. Due to the continuous variation of the constituents, the properties such as Young modulus, Poisson ratio, shear modulus, material density, and coefficient of thermal expansion vary smoothly and continuously in preferred directions [8]. Therefore, high values of shear stresses that may be induced in laminated composites with substantial difference in material properties are prevented. FGMs have received many applications in various engineering fields including mechanical, civil, aerospace, and chemical engineering [9]. As for the research work related to biosensors, Rahimi and Davoodinik [10] developed analytical and adomian decomposition methods to determine the large defection of functionally graded (FG) cantilever flexible beams under inclined end loading by fully accounting for geometric nonlinearities and incorporating the physical properties of FGMs. It is found that the adomian decomposition method is useful toward the design of FG compliant mechanisms driven by smart actuators. Many researchers analyzed microbeams made of FGM using the modified couple stress theory [11-15]. For example, Asghari et al. [11, 12] presented size-dependent formulations and investigated the static and free vibration behaviors in the elastic range of both FG Euler-Bernoulli and FG Timoshenko beams in which properties vary according to a power law in the thickness direction. They found that modeling beams on the basis of non-classic theories, such as the couple stress theory, lead to more stiffness, whereas modeling based on the classical continuum theory underestimates the stiffness especially when the beam thickness is relatively small. Also, Farokhi et al. [13] proposed the first study to examine the size-dependent nonlinear dynamics of an FG microcantilever undergoing large oscillations. They obtained the resonances highlighting the influence of different microsystem parameters including the material gradient index. In addition, Rokni et al. [14] analytically investigated the free vibration behavior of cantilever beams made of a polymer nanocomposite reinforced by multi-walled carbon nanotubes. They considered continuous variations of the mechanical properties of the nanocomposite in the longitudinal direction of the beam. Furthermore, Rahi [15] investigated the dynamic response of multiple-layer microcantilever beams. It is found that the length, width, and thickness of each layer are extremely effective on the vibration characteristic of the microbeams.

The magnitude of surface stresses induced in microcantilever-based biosensors is very small, and consequently, it is of great importance to enhance the performance of these sensors, including sensitivity. This can be achieved by proper choice of the materials (which in turn changes Young modulus), surface properties, and geometric parameters [16]. Therefore, this paper aims to properly design microcantilever-based biosensors with high sensitivity for detecting various diseases by controlling the deflection through continuous gradation of various geometrical and material parameters. These parameters, which vary in the longitudinal direction of the sensor according to a power law, include thickness of the sensor and Young modulus of the material. The model developed by Zhang et al. [6] is applied while some modifications are considered to account for the present analyses of varying the considered parameters. The effects of the exponent of the gradation function as well as the ratio between the parameter at the free and fixed ends on the maximum deflection, which occurs at the free end of the sensor, are investigated. In addition, the equivalent force causing the deflection is also evaluated using Castigliano method for two cases. The first case accounts for a concentrated force at the free end while the second case considers a distributed load along the biosensor. 


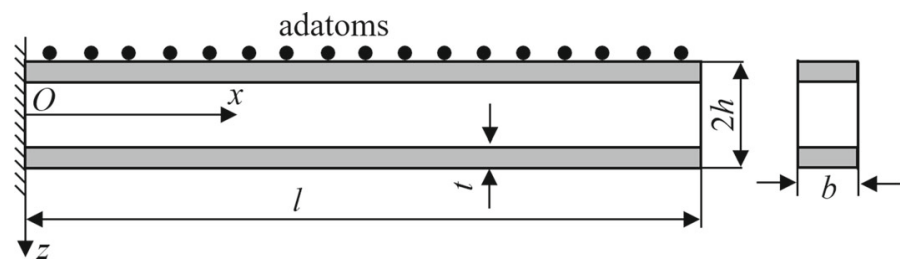

Fig. 1 The considered microcantilever biosensor with adsorbates on its upper surface

\section{Governing equations}

There are many types of biosensors, which are extremely versatile when classified according to the working mechanism such as the detector, the transduction, and application sensors [2]. The mechanical transduction mechanism of biosensors is based on the bending of microfabricated cantilever caused by the adsorption of, for example, molecules onto the surface [6]. The considered biosensor is modeled as a microcantilever beam with adsorbed atoms on its upper surface as shown in Fig. 1. The adsorbed molecules are modeled as a layer with a finite thickness. The length, thickness, and width of the microcantilever are $l, 2 h$, and $b$, respectively. On the other hand, $t$ is the thickness of each of the top and bottom surface layers.

Figure 2a shows the considered biosensor before bending or deflection where the adatoms statistically uniformly distribute on the surface and the distance between two adjacent adatoms $P$ and $Q$ equals $d$. This distance is controlling the adsorption density $\eta$ where $d=\sqrt{1 / \eta}$. Also, the distance between two adjacent atoms ( 1 and 2 or 3 and 4 ) on the substrate surface is $c$. When the chemical reaction occurs, force and therefore surface stress are induced causing deflection or bending of the biosensor as depicted in Fig. 2b. It is assumed that the curvature of the bent beam is infinitesimal and uniform along the beam length and that the dimension $a$ keeps constant with the cantilever bending. The distance $f$ is the increase of the spacing between any two adjacent surface atoms due to bending. Therefore, the initial spacing $c$ becomes $c+f$ after bending. Also, the increase of the spacing between any two adjacent adatoms due to bending is $e$. The energy method developed by Zhang et al. [6] is modified to be valid for the continuous variation of the considered shape and material parameters and then applied to calculate the deflection of the beam. The total free energy of the system is composed of three parts. These are the van der Waals interaction potential energy between the adsorbates and the substrate, van der Waals interaction potential energy among the adsorbates, and the elastic strain energy of the bending cantilever.

\subsection{Interaction energy between adatoms and substrate}

The well-known Lennard-Jones potential can be used to evaluate the van der Waals pair potential between any two arbitrary atoms as follows

$$
U^{v d W}(r)=-\frac{A_{\propto}}{r^{6}}+\frac{B_{\propto}}{r^{12}}
$$

where $r$ is the distance between two atoms and $A_{\alpha}$ and $B_{\alpha}$ are the Lennard-Jones constants. $\alpha=1$ stands for the interaction between an adatom and a substrate atom whereas $\alpha=2$ stands for the interaction between two adatoms.

The increase of the interspacing between two adjacent surface atoms due to bending is given by

$$
f=h \frac{c}{R}
$$

where $R$ is the radius of curvature of the cantilever.

The distance between an adatom $P$ and substrate atom 1 (or between $Q$ and 3 ) is given by

$$
d_{P 1}=d_{Q 3}=\sqrt{a^{2}+\left(\frac{c+f}{2}\right)^{2}}
$$




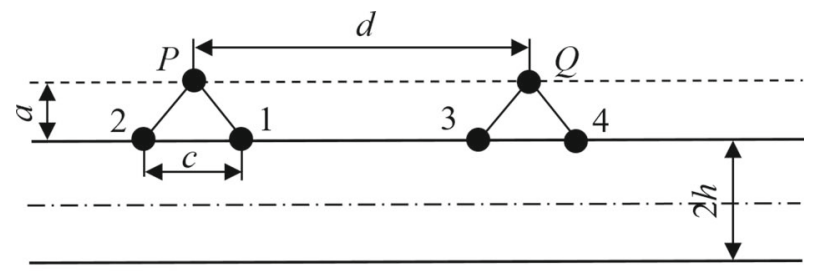

(a)

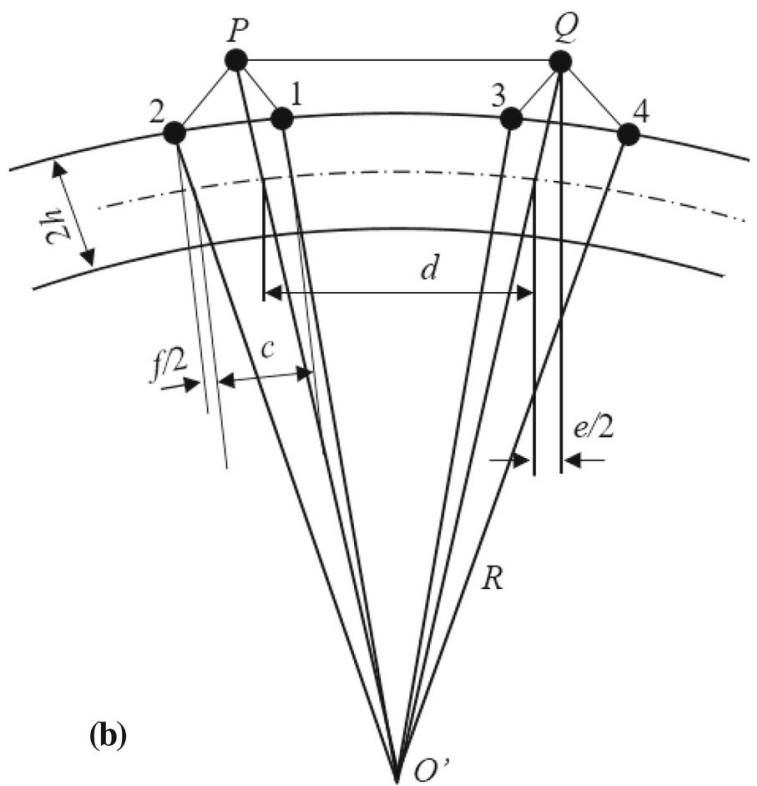

Fig. 2 Positions of the distributed adatoms and substrate surface atoms. a Before bending. b After bending

Thus, the van der Waals interaction energy between adatom $P$ and substrate atom 1 (or between $Q$ and 3 ) can be written as

$$
U_{P 1}^{v d W}=U_{Q 3}^{v d W}=-\frac{A_{1}}{\left[a^{2}+\frac{1}{4} c^{2}(1+h k)^{2}\right]^{3}}+\frac{B_{1}}{\left[a^{2}+\frac{1}{4} c^{2}(1+h k)^{2}\right]^{6}}
$$

where $k=1 / R$ denotes the curvature of the biosensor.

Under bending, the distance between two neighboring adatoms $P$ and $Q$ changes to

$$
d_{P Q}=d+e
$$

where

$$
e=(h+a) \frac{d}{R}
$$

Then, the van der Waals interaction energy between adatoms $P$ and $Q$ is given by

$$
U_{P Q}^{v d W}=-\frac{A_{2}}{d^{6}[1+(h+a) k]^{6}}+\frac{B_{2}}{d^{12}[1+(h+a) k]^{12}}
$$

The total interaction energy between the adatoms and substrate is expressed by integration as

$$
U_{\text {int }}=\int_{0}^{l} u_{\text {int }} \mathrm{d} x
$$


where

$$
u_{\mathrm{int}}=\eta b\left(U_{P Q}^{v d W}+U_{P 1}^{v d W}+U_{Q 3}^{v d W}\right)
$$

In the case of small curvature, $u_{\mathrm{int}}$ can be approximated by the first three terms of its Taylor series with respect to the initial, undeformed, state as,

$$
u_{\mathrm{int}}=\left.u_{\mathrm{int}}\right|_{\kappa=0}+\left.\frac{\partial u_{\mathrm{int}}}{\partial \kappa}\right|_{\kappa=0} \kappa+\left.\frac{1}{2} \frac{\partial^{2} u_{\mathrm{int}}}{\partial \kappa^{2}}\right|_{\kappa=0} \kappa^{2}
$$

\subsection{Elastic strain energy}

The considered microcantilever sensor or also known as surface stress sensor is categorized under the micromechanical-based sensors. The cantilever can be divided into three laminated layers, including two identical surface layers of the same thickness $t$ and a bulk layer of thickness $2 h-2 t$, as shown in Fig. 1 . Though the elastic strain energy is composed of two parts due to both bending and axial loads, the elastic strain energy due to axial loading can be neglected [1]. The relaxed surface layers and the bulk layer are assumed to be made of different homogeneous materials possessing average Young moduli of $E_{t}$ and $E$, respectively. Therefore, the surface elastic constant can be given as

$$
S=t\left(E_{t}-E\right)
$$

The residual surface stress can be written as

$$
\gamma_{0}=t \sigma_{0}
$$

where $\sigma_{0}$ is the uniform stress of the surface layer.

Under the assumption of small deflection, the strain in the longitudinal direction of the cantilever beam is given by

$$
\varepsilon_{x}=-\mathcal{Z} \kappa
$$

Thus, the longitudinal induced stresses in the bulk, $\sigma_{x}^{c}$, and the surface layers, $\sigma_{x}^{s}$, can be evaluated by

$$
\begin{gathered}
\sigma_{x}^{c}=-\mathrm{E} \mathcal{Z}_{\kappa}, \\
\sigma_{x}^{s}=\sigma_{0}-\mathrm{E} \mathcal{Z}_{\kappa},
\end{gathered}
$$

Then, the elastic strain energies of the bulk $U_{c}$ and the surface layers $U_{s}$ are written as [17]:

$$
\begin{gathered}
U_{c}=\frac{1}{2} E I_{c} \int_{0}^{l} \kappa^{2} \mathrm{~d} x, \\
U_{s}=\frac{1}{2} E_{S} I_{s} \int_{0}^{l} \kappa^{2} \mathrm{~d} x,
\end{gathered}
$$

where $I_{c}$ and $I_{s}$ are the area moment of the inertia of the bulk and surface layers, respectively, and they are given by

$$
\begin{gathered}
I_{c}=\frac{2}{3} b h^{3}\left(1-\frac{t}{h}\right)^{3} \\
I_{s}=2 b t h^{2}\left[1-\frac{t}{h}+\frac{1}{3}\left(\frac{t}{h}\right)^{2}\right]
\end{gathered}
$$


Due to symmetry of the cross section, the contributions of the upper and lower surfaces to the total strain energy cancel each other. Therefore, the total elastic strain energy of the bending cantilever is independent on the surface stress and is written as

$$
U_{b}=U_{c}+U_{s}=\frac{1}{2} E^{*} I \int_{0}^{l} \kappa^{2} \mathrm{~d} x,
$$

where $E^{*} I$ denotes the effective bending stiffness of the cantilever and is given by

$$
E^{*} I=E I+2 S b h^{2}\left[1-\frac{t}{h}+\frac{1}{3}\left(\frac{t}{h}\right)^{2}\right],
$$

and $I=2 b h^{3} / 3$ denotes the area moment of the inertia of the rectangular beam.

\subsection{Cantilever deflection}

Upon adsorption, the total potential energy of the adsorbate-cantilever system is expressed as

$$
\Pi=U_{b}+U_{\mathrm{int}}
$$

By minimizing the total potential energy according to the variational principle and by using the following approximate relation:

$$
\kappa=\frac{\partial^{2} w}{\partial x^{2}}
$$

the deflection curve is derived as

$$
\frac{\partial^{4} w}{\partial x^{4}}=0
$$

The corresponding boundary conditions of the microcantilever are that each of the deflection and slope at the support and the shear force and bending moment at the free end equals zero, i.e.,

$$
\begin{aligned}
& \left.w\right|_{x=0}=0,\left.\quad \frac{\partial w}{\partial x}\right|_{x=0}=0, \\
& \left.\frac{\partial^{2} w}{\partial x^{2}}\right|_{x=l}=-\left.\frac{\partial u_{\text {int }}}{\partial k}\right|_{\kappa=0}\left(E^{*} I+\left.\frac{\partial^{2} u_{\text {int }}}{\partial k^{2}}\right|_{\kappa=0}\right)^{-1},\left.\quad \frac{\partial^{3} w}{\partial x^{3}}\right|_{x=l}=0 .
\end{aligned}
$$

If the cantilever has a uniform cross section and its material is homogenous, isotropic, and obeys Hooke's law, Eqs. (24) and (25) lead to the deflection curve of the cantilever beam as

$$
w(x)=-\left.\frac{1}{2} \frac{\partial u_{\text {int }}}{\partial k}\right|_{\kappa=0}\left(E^{*} I+\left.\frac{\partial^{2} u_{\text {int }}}{\partial k^{2}}\right|_{\kappa=0}\right)^{-1} x^{2} .
$$

Therefore, the deflection at the free end of the cantilever $\left(w_{l}\right)$ can be obtained by substituting $x=l$ and is given by

$$
w_{l}=-\left.\frac{1}{2} \frac{\partial u_{\text {int }}}{\partial k}\right|_{\kappa=0}\left(E^{*} I+\left.\frac{\partial^{2} u_{\text {int }}}{\partial k^{2}}\right|_{\kappa=0}\right)^{-1} l^{2}
$$

The evaluated deflection at the free end of the beam can be used to calculate the induced surface stress [18], as

$$
w_{l}=\sigma l^{2}(1-v) / E h^{2}
$$

the induced force is given as a function of the surface stress as

$$
F=2 \sigma b h(1-v) / l
$$


It can also be given as a function of the deflection at the free end as

$$
F=2 E b w_{l}(h / l)^{3}
$$

In addition, the induced force can be evalauted by applying the double integration method or Castigliano method, which is based on the energy approach [19], as

$$
\begin{gathered}
w_{l}=\iint M \mathrm{~d} x \text { at } x=l \quad \text { (For the double integration method) } \\
w_{l}=\int_{0}^{l} \frac{M}{E I} \frac{\partial M}{\partial F} \mathrm{~d} x, \quad \text { (For Castigliano method) }
\end{gathered}
$$

where $M$ is the bending moment at a distance $x$ from the fixed end, $F$ is the concentrated force at the point where the deflection $w_{l}$ occurs that represents the free end in the current study.

Two cases are considered: Case 1 accounts for a concentrated force at the free end of the beam. Since the chemical reaction is supposed to occur along the beam, Case 2 accounts for a distributed load along the biosensor.

Case 1 Applying Castigliano method, the concentrated force $F$ at the free end of the beam can be evaluated as

$$
F=\frac{w_{l}}{\int_{0}^{l} \frac{(l-x)^{2}}{E I} \mathrm{~d} x}
$$

Since $E$ and $I$ are considered to vary with the position $x$, they are included in the integration.

Case 2 The distributed load $q$ along the beam can be evaluated as

$$
q=\frac{w_{l}}{\int_{0}^{l} \frac{\left(l x-x^{2} / 2-l^{2} / 2\right)(x-l)}{E I} \mathrm{~d} x}
$$

For the same value of the deflection at the free end, it is known that the ratio between the concentrated force at the free end and the distributed load equals $3 l / 8$ when $E$ and $h$ are constant along the beam [19].

\subsection{Functionally graded biosensor}

The functionally graded (FG) concept implies that one or more parameters vary continuously with the position according to a certain function. In the current study, the thickness as a geometrical parameter and Young modulus as a material parameter are considered to vary continuously along the length of the biosensor according to a power law [20]. At any position $x$, the value of each of these parameters $(P(x))$ is written as

$$
P(x)=P_{o}+\left(P_{1}-P_{o}\right)\left(\frac{x}{l}\right)^{n p}
$$

where $P_{o}$ and $P_{1}$ are the values at the fixed and free ends, respectively. Also, $n_{p}$ is an exponent.

Therefore, a nondimensional form of a considered parameter is given by

$$
\frac{P(x)-P_{o}}{P_{1}-P_{o}}=\left(\frac{x}{l}\right)^{n_{p}}
$$

When introducing the nondimensional position $\mathrm{X}\left(=\frac{x}{l}\right)$, Eq. (36) can be written as

$$
\frac{P(x)-P_{o}}{P_{1}-P_{o}}=(X)^{n_{p}}
$$

If Young modulus $(E)$, for example, is considered, Eq. (37) is written as

$$
\frac{E(x)-E_{o}}{E_{1}-E_{o}}=(X)^{n_{E}}
$$

Figure 3 shows the variation of any of these nondimensional parameters along the beam for various values of the exponent $n_{p}$. When $n_{p}=1$, the parameter changes linearly from the fixed end to the free end. When $n_{p}$ 


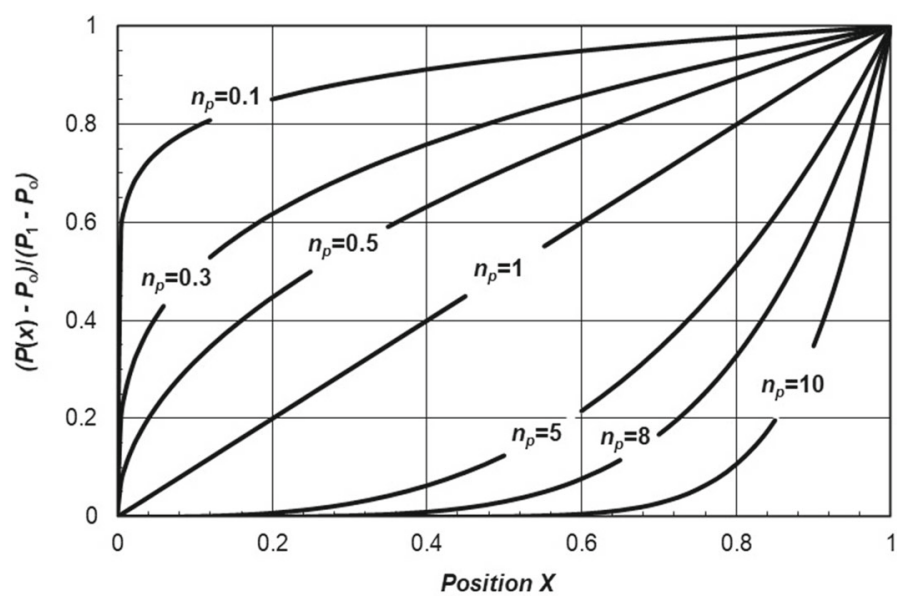

Fig. 3 Variations of the parameters according to the power law

$=0.1$, the parameter changes sharply at the fixed end but smoothly at the free end of the biosensor and the main part of the beam has values close to that at the free end. On the other hand, when $n_{p}=10$, the parameter behavior is opposite to that of $n_{p}=0.1$. For the sake of clarification, if the considered parameter is Young modulus and the fixed end is more compliant than the free end, the beam with low $n_{p}$ is stiffer than that of high $n_{p}$. Also, when the considered parameter is the thickness of the beam and the free end is thinner than the fixed end, high $n_{p}$ results in more rigid beam than that of low $n_{p}$. That is due to the higher effective moment of inertia of the cross section when $n_{p}$ is high.

All relations of the previous subsections, dealing with evaluating the deflection and force, should include the power laws of the thickness and Young modulus, and therefore integration should be carried out whenever required.

\section{Results and discussions}

In order to perform numerical calculations and obtain results, the values of different parameters considered by Zhang et al. [6] for the case of adsorbed mercury $(\mathrm{Hg})$ on gold $(\mathrm{Au}(100))$ surface are taken into account, unless otherwise mentioned. The length, width, and thickness of the beam are 180, 18, and $0.6 \mu \mathrm{m}$, respectively. Also, the material parameters, which include Young modulus and Poisson ratio, of the homogeneous beam are $78.6 \mathrm{GPa}$ and 0.42 , respectively. For the beams made of functionally graded materials or when varying Young modulus and/or the thickness along the beam, the above mentioned values are considered at the fixed end of the beam. Also, it is assumed that all of the considered biosensors are made of isotropic materials. In addition, the concentration of the virus on the beam surface is referred to the nondimensional or relative adsorption density $\eta_{r}=\eta / \eta_{m}$ where $\eta_{m}=1 / c^{2}$, which reflects how much the quantity of the virus interacted with the cantilever surface. Therefore, $\eta_{r}$ ranges from zero to one, where one indicates that the whole surface of the cantilever is covered with the virus whereas zero indicates that there is no virus exists on the surface.

In some engineering designs, it is recommended that the design parameters vary linearly with the position [20]. Therefore, it is important to have some insights into the effects of such variations on the beam deflection. Figure 4 shows the variation of the deflection at the free end of the beam $\left(w_{l}\right)$ with the relative adsorption density $\left(\eta_{r}\right)$ for various ratios of the thickness at the free end to that at the fixed end $\left(R_{h}\right)$ when the thickness exponent $n_{h}=1$. When one side of the microcantilever is functionalized with bioreceptors for detecting an analyte, surface stress is generated when the target analyte adsorbs onto the surface. The difference in surface stress between the top and bottom surfaces of the microcantilever produces bending. The bending can be upwards or downwards depending on the type of the molecular interactions. It can be noted from Fig. 4 that there is a critical value of $\eta_{r}$ which corresponds to a conversion of the bending direction of the cantilever. For all values of $R_{h}$, it can be seen that $w_{l}$ is positive or in other words the beam bends downwards provided that $\eta_{r}$ less than the critical value. Beyond this value, $w_{l}$ is negative or in other words the beam bends up. The bend-up beams have, in general, higher $w_{l}$ and hence high sensitivity, especially when $\eta_{r}=1$, compared to the bend down beams. Therefore, based on the point of view of measuring $w_{l}$, it may be convenient to choose 


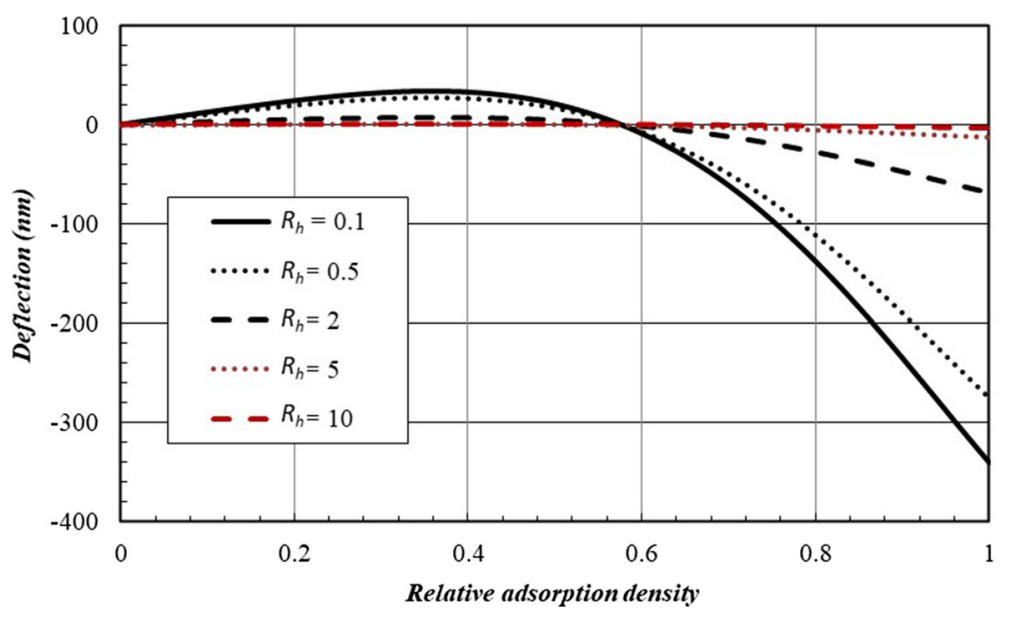

Fig. 4 Variation of $w_{l}$ with the relative adsorption density for various values of $R_{h}$ when $n_{h}=1$

the conditions of bend-up beams for biosensors. Furthermore, maximum $w_{l}\left(w_{\max }\right)$ logically occurs when $\eta_{r}=1$ for all values of $R_{h}$. That is because when the adsorption density increases, which means the virus concentration on the cantilever surface is high, the induced surface stress increases due to the more effective chemical reaction between the cantilever surface and the virus. However, $w_{\max }$ is inversely proportional to $R_{h}$ due to the increase of the moment of inertia of the cross section, which leads to an increase of the beam rigidity. From the practical point of view, it may be recommended to use beams with low values of $R_{h}$ as the deflection is easier to be measured. However, reasonable $R_{h}$ can be chosen for a proper biosensor with reasonable sensitivity for a certain field of application.

The thickness may vary nonlinearly, and this can be attained by varying the thickness exponent $\left[n_{h}\right.$ in Eq. (37)]. Figure 5 depicts the variation of $w_{l}$ with $\eta_{r}$ for various values of $n_{h}$ when $R_{h}=0.5$. It can be seen that $w_{\max }$ is inversely proportional to $n_{h}$ because of the direct proportion of the effective moment of inertia of the cross section and consequently, the beam rigidity with $n_{h}$. This can be explained by referring to Fig. 3, when $n_{h}=0.1$, the main part of the cantilever has thickness close to that at the free end. On the contrary, the main part of the cantilever has thickness close to that at the fixed end when $n_{h}=10$. It is important to mention that the deflection values approach those of Zhang et al. [6] at the highest value of $n_{h}$ whereas they are the same when $n_{h}=\infty$. This good agreement of the results verifies the validity of the current analyses for predicting the behaviors of FG micro-biosensors. However, $n_{h}=0.1$ and 10 may be not reasonable from the practical point of view as the thickness is about uniform along the beam except very small regions. It is found from other calculations that when $R_{h}>1$, the dependency of the deflection on $n_{h}$ is opposite to that shown in Fig. 5 and that is because of the beam rigidity. Eventually, the obtained results can be used for enhancing the performance parameters of biosensors such as decreasing the limit of detection.

Functionally graded materials are characterized by continuous variations of their properties with the position. In the present analyses, the main material property is Young modulus $(E)$. Therefore, it is of vital importance to discuss the effects of varying $E$ in the longitudinal direction of the beam on $w_{l}$, and that is depicted in Fig. 6 considering $n_{E}=1$. Since $R_{E}$ represents the ratio of $E$ at the free end to that at the fixed end, the free end is more compliant/(stiffer) than the fixed end when $R_{E}<1 /\left(R_{E}>1\right)$. It can be seen that $w_{\max }$, which occurs at $\eta_{r}=1$ for any value of $R_{E}$, is inversely proportional to $R_{E}$ because of the direct proportion of the beam effective stiffness with $R_{E}$ and the inverse proportion of the deflection with stiffness. Hence, from the practical point of view and based on the measurement perspectives and sensitivity, it may be recommended to use beams with low values of $R_{E}$. Furthermore, reasonable $R_{E}$ can be chosen for manufacturing biosensors with low limit of both blank and detection.

The effects of the stiffness exponent $\left(n_{E}\right)$ on $w_{l}$ are shown in Fig. 7 when $R_{E}=0.5$. It can be seen that $w_{\max }$ is inversely proportional to $n_{E}$ because of the direct proportion of the beam equivalent stiffness with $n_{E}$ as explained earlier (Fig. 3). In addition, the results approach those of Zhang et al. [6] at the highest value of $n_{E}$. It is found from other calculations that when $R_{E}>1$, the dependency of $w_{\max }$ on $n_{E}$ is opposite to that shown in Fig. 7 due to the variation of the effective stiffness.

It is important to have some insight into the behavior of $w_{l}$ when varying $E$ and $h$ together and to compare the results with those of individual variations as well as those of Zhang et al. [6] who considered uniform 


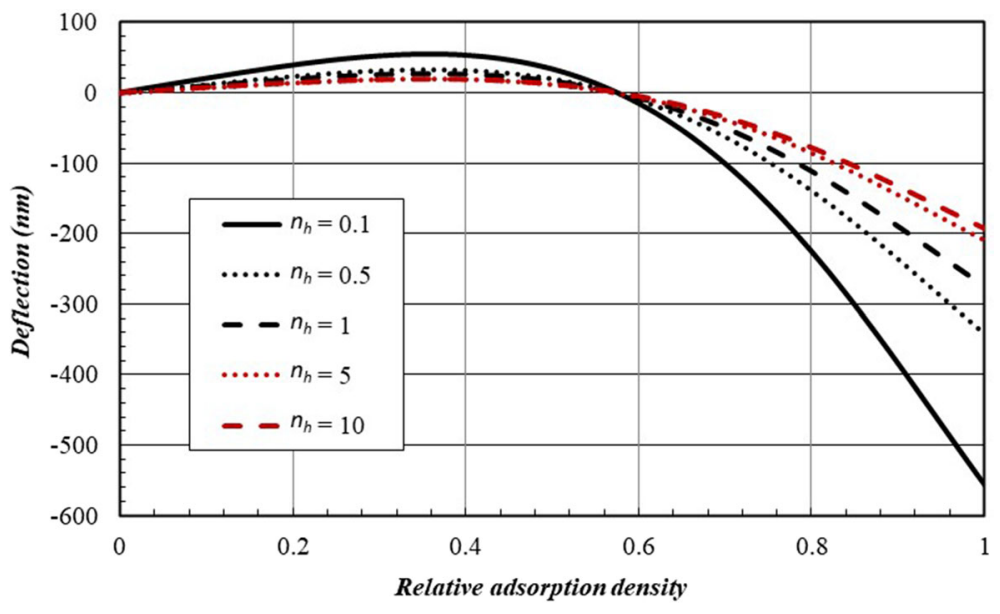

Fig. 5 Variation of $w_{l}$ with the relative adsorption density for various values of $n_{h}$ when $R_{h}=0.5$

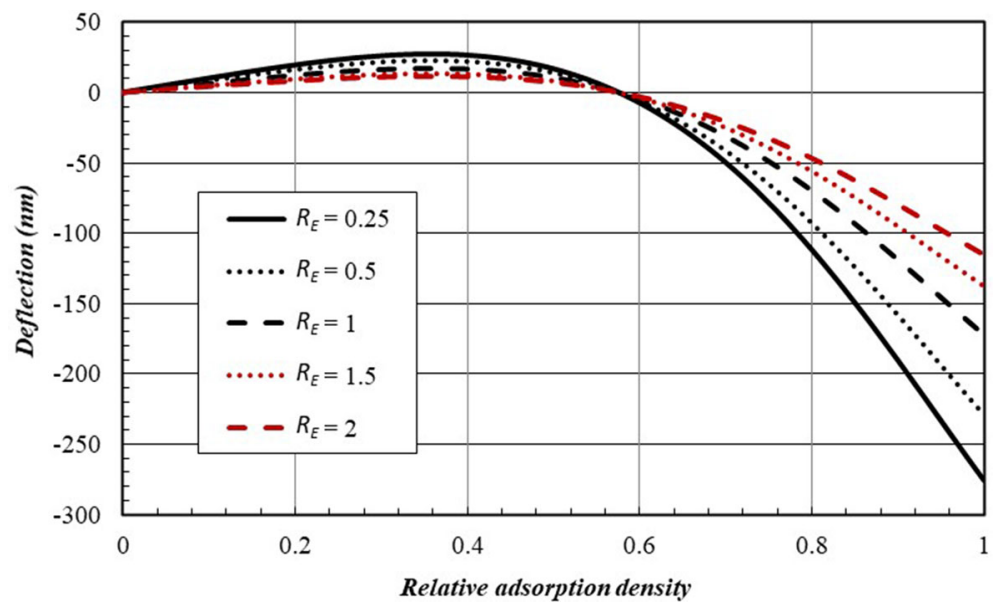

Fig. 6 Variation of $w_{l}$ with the relative adsorption density for various values of $R_{E}$ when $n_{E}=1$

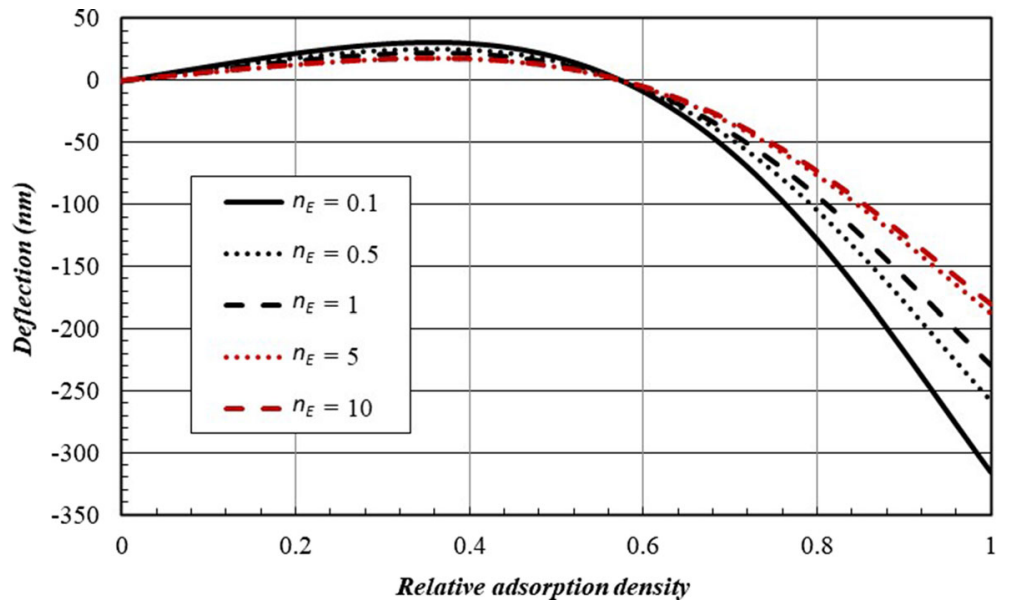

Fig. 7 Variation of $w_{l}$ with the relative adsorption density for various values of $n_{E}$ when $R_{E}=0.5$ 


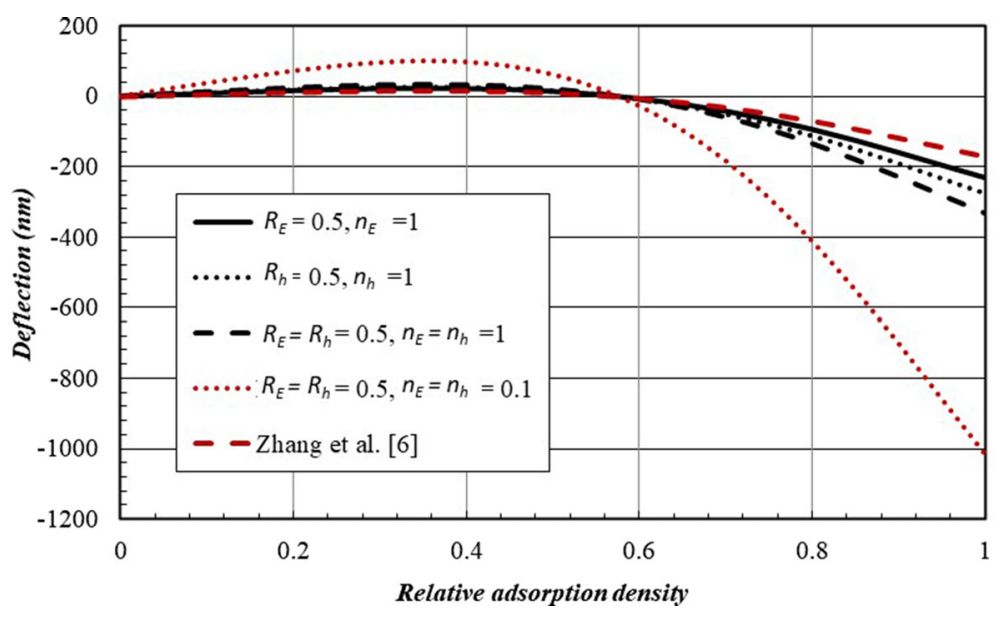

Fig. 8 Variation of $w_{l}$ with the relative adsorption density when varying the thickness and stiffness of the beam

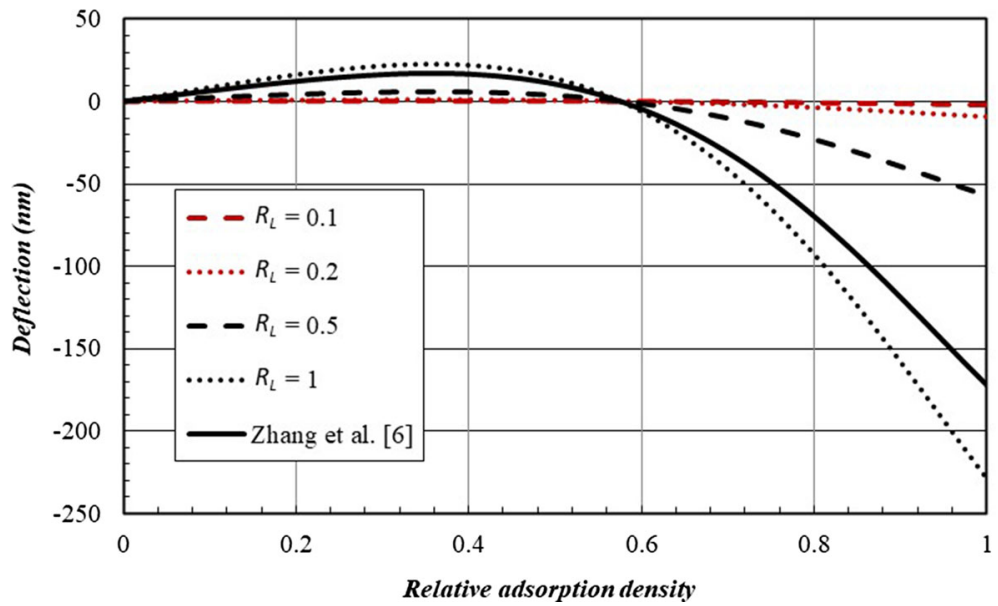

Fig. 9 Variation of $w_{l}$ with the relative adsorption density for various values of beam length when $R_{E}=0.5$ and $n_{E}=1$

and homogenous beams. It can be seen from Fig. 8 that when $R_{E}=0.5, w_{\max }$ is higher than that of Zhang et al. [6] due to the stiffness reduction. Also, the individual reduction of $h\left(R_{h}=0.5\right)$ leads to a reduction of the moment of inertia of the cross section which results in a reduction of the beam rigidity and hence $w_{\max }$ increases. However, the individual reduction of $h$ affects the deflection more than that of $E$. When the thickness and stiffness are reduced together $\left(R_{E}=R_{h}=0.5\right)$, the increased amount of $w_{\max }$ is more than the summation of the individual reductions. Therefore, the superposition concept is not applicable in this case. On the other hand, to investigate the effects of the exponents, a beam with $R_{h}=R_{E}=0.5$ and $n_{E}=n_{h}=0.1$ is considered, and it can be seen that $w_{\max }$ increases due to the decrease of both the stiffness and rigidity as explained earlier. Furthermore, Fig. 8 reveals that all of the considered FG beams logically have higher values of $w_{\max }$ than those of the homogeneous and uniform beam of Zhang et al. [6], that positively contribute to enhancing the biosensor performance parameters such as sensitivity.

Figure 9 depicts the variation of $w_{l}$ for different values of the beam length when $R_{E}=0.5$ and $n_{E}=1$ while $R_{L}$ is the ratio of the beam length to that considered by Zhang et al. [6]. It can be seen that at the same relative adsorption density, or in other words at the same surface stress due to chemical reaction between the virus and the cantilever, $w_{\max }$ is logically increasing with $R_{L}$. Also, it is worthy to mention that $w_{\max }$ is decreased by $75 \%$ when $R_{l}$ equals 0.5 which is consistent with Eq. (27). It is found from the numerical results that other values of $R_{L}$ lead to the same proportionality rule with the beam length. Eventually, the beam length can be properly chosen in order to have an easy-to measure $w_{\max }$.

Figures $4,5,6,7,8$, and 9 can be used for tailoring the shape and material of microcantilever-based biosensors to satisfy specific constraints for certain application and for detecting various diseases. Since $w_{\max }$ 


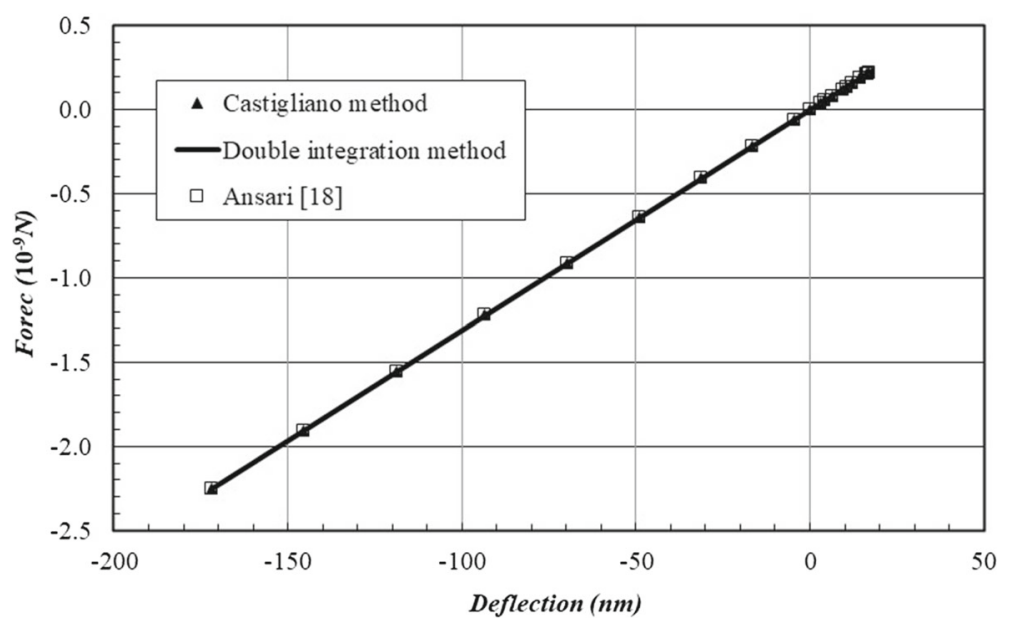

Fig. 10 The predicted concentrated force using different methods

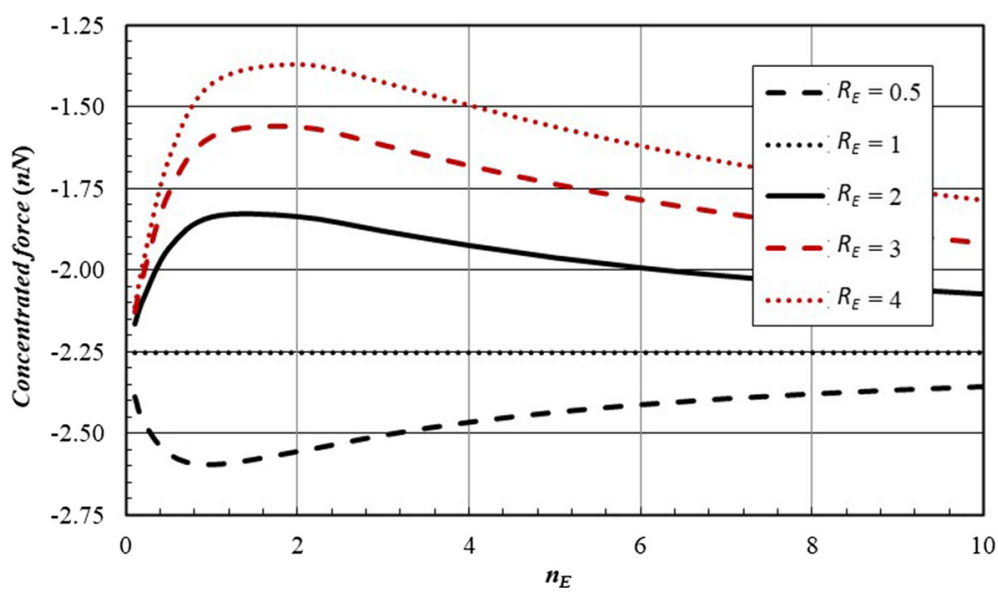

Fig. 11 Variation of the concentrated force with the exponent $n_{E}$ of the power law for various values of $R_{E}$

depends greatly on $n_{E}, n_{h}, R_{E}, R_{h}, R_{L}, E_{o}$, and $h_{o}$, biosensors with high sensetivity and accuracy, and low limits of both blank and detection can be designed by proper selection of these parameters. However, the width $b$ of the biosensor is not affecting the deflection [6]. In addition, biosensors with high signal-to-noise ratio, which represent the input to the the measuring technique, can be attained for different applications as the measured deflection value can be predefined.

For the design purpose and to avoid fracture of biosensors, it is important to shed some light on the induced force. Applying the elasticity principles, the concentrated force at the free end causing $w_{\max }$ is evaluated using the Castigliano and double integration methods [19], and the method given in [18]. Figure 10 shows that the force is logically increasing linearly with $w_{\max }$ for all methods. Also, it can be seen that the results of these three methods are similar.

Applying Castigliano method, the variation of the induced concentrated force at the free end causing $w_{\max }$ is plotted versus $n_{E} /\left(n_{h}\right)$ for various values of $R_{E} /\left(R_{h}\right)$ in Fig. 11/(Fig. 12), respectively. It can be seen that the force is logically constant for different values of the exponent $n_{E} /\left(n_{h}\right)$ when $R_{E} /\left(R_{h}\right)$ equals 1 because $E /(h)$ in this case is the same all over the beam. Also, at the same $n_{E} /\left(n_{h}\right)$, the force magnitude is inversely proportional to $R_{E} /\left(R_{h}\right)$. When $R_{E} /\left(R_{h}\right)>1$, the induced force initially decreases with $n_{E} /\left(n_{h}\right)$ to a certain limit after which the force increases. However, the decreasing rate is much higher than the increasing rate that is very slight for $n_{h}$. Also, the behavior of the force magnitude when $R_{E} /\left(R_{h}\right)<1$ is opposite to that of $R_{E} /\left(R_{h}\right)>1$.

Since the intermolecular reaction between the adsorbates and the substrate atoms occurs along the beam length, it may be close to reality to consider the distributed load, which acts all over the beam, causing the deflection at the free end. The effects of the exponents $n_{E}$ and $n_{h}$ on the induced distributed load for various values of $R_{E}$ and $R_{h}$ are depicted in Figs. 13 and 14, respectively. As can be seen, the behavior of the distributed 


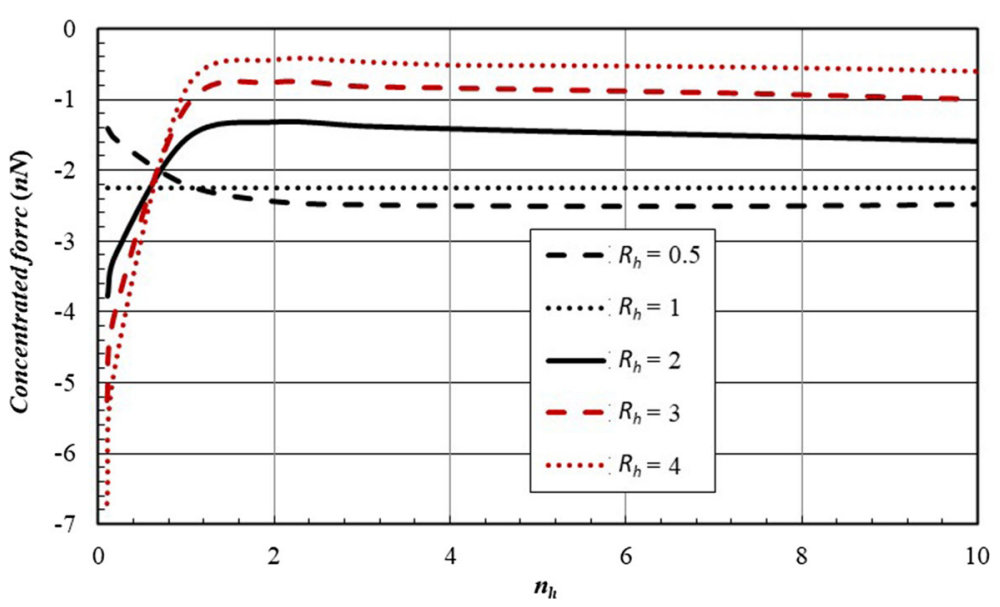

Fig. 12 Variation of the concentrated force with the exponent of the power law $n_{h}$ for various values of $R_{h}$

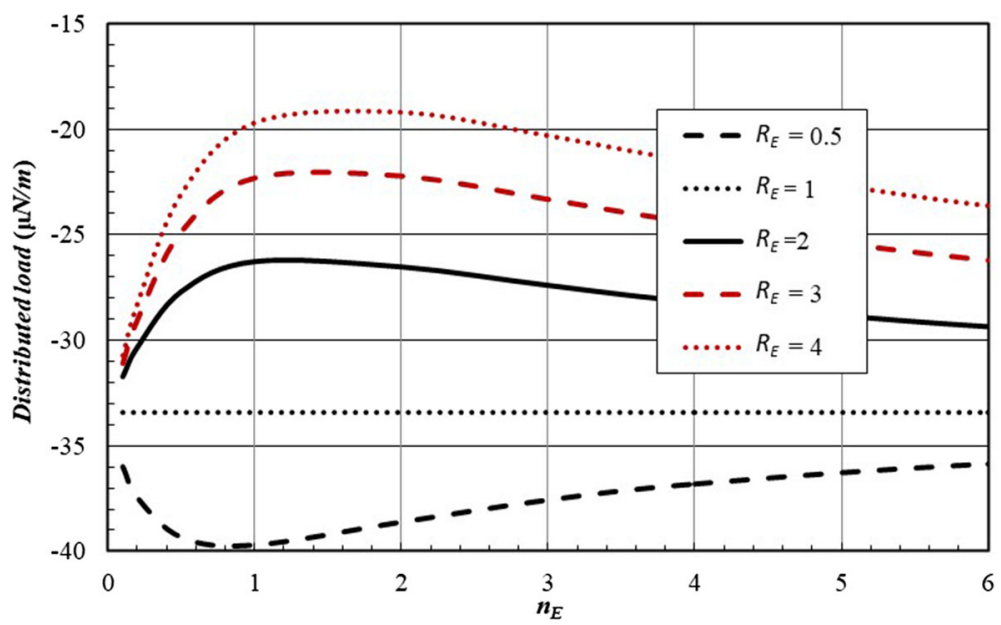

Fig. 13 Variation of the distributed load with the exponent $n_{E}$ of the power law for various values of $R_{E}$

load is almost the same as that of the concentrated force (Figs. 11 and 12). However, the distributed load is four order of magnitude higher than the concentrated force. Most importantly, it is found when $R_{E}=1 /\left(R_{h}=1\right)$ that the ratio between the concentrated force at the free end (Fig. 11/Fig. 12) and the corresponding distributed load (Fig. 13/Fig. 14) equals $3 l / 8$ [19]. That is because $E$ and $h$, in this case, are not varying in the longitudinal direction of the beam.

\section{Conclusions}

The effects of various functionally graded parameters on the behavior of microcantilever-based biosensors are investigated for the sake of enhancing the performance, which includes sensitivity and accuracy, for detecting various diseases. These parameters are related to the material and shape and vary gradually and continuously according to a power law. The influences of the surface stress, van der Waals intermolecular forces, and the total energy of the beam are taken into consideration. Numerical methods, which overcome the complexity due to size dependency and gradation of the considered parameters, are applied. It is found that the exponent of the gradation function, Young modulus, thickness, and length of the sensor play important roles in the mechanical bending that is represented by the deflection at the free end of the sensor. For example, the deflection is directly proportional to the length and the percent reduction of each of the thickness and Young modulus at the free end relative to those at the fixed end. This is attributed to the decrease of both the moment of inertia of the cross section and the beam effective stiffness. Also, the deflection is inversely proportional to the exponent of 


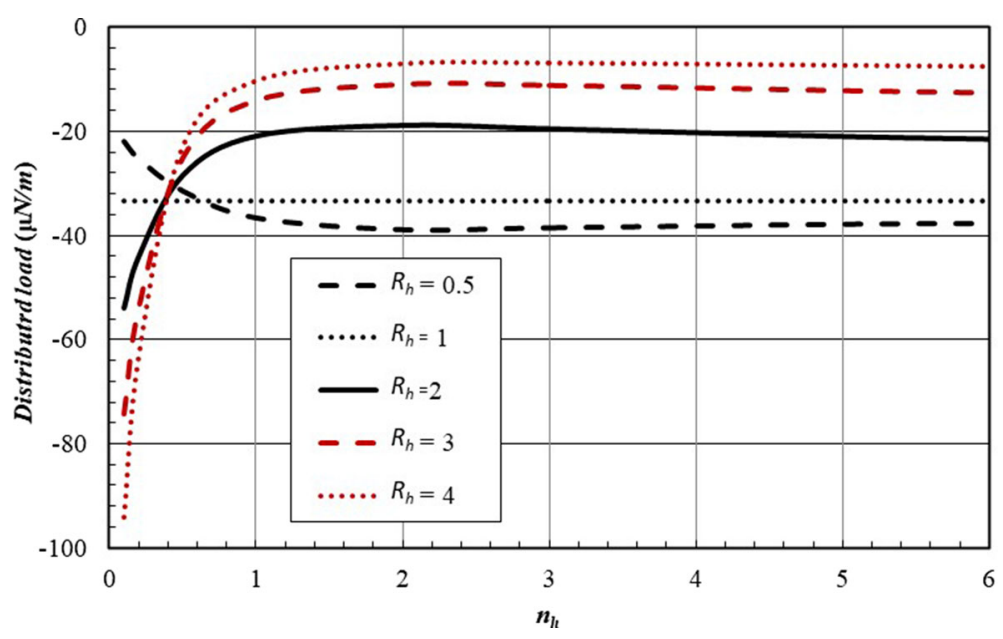

Fig. 14 Variation of the distributed load with the exponent of the power law $n_{h}$ for various values of $R_{h}$

the gradation function. However, if the beam is thinner or more compliant at the fixed end, the deflection is directly proportional to the exponent of the gradation function.

This study is helpful for tailoring the shape and material of a microcantilever-based biosensor for detecting various diseases as the measurable deflection range can be predefined to satisfy specific constraints for certain application. Therefore, biosensors with enhanced performance, which include sensetivity, and limits of both blank and detection of viruses can be attained. Also, designing biosensors with high signal-to-noise ratio, which is essential for the measurement techniques, can be achieved by applying the current analyses.

\section{Compliance with ethical standards}

Conflict of interest On behalf of all authors, the corresponding author states that there is no conflict of interest.

\section{References}

1. Stoney, G.G.: The tension of metallic films deposited by electrolysis. Proc. R. Soc. Lond Ser. A Contain. Pap. Math. Phys. Character 82, 172-175 (1909)

2. Raiteri, R., Grattarola, M., Butt, H.J., Skadal, P.: Micromechanical cantilever-based biosensors. Sensors Actuators B Chem. 79, 115-126 (2001)

3. Zhao, Y.: Surface stress detection and mechanism study with microcantilever based sensor for biomolecular monolayers. $\mathrm{Ph} . \mathrm{D}$. thesis, Iowa University (2014)

4. Lim, Y.C., Kouzani, A., Wei, D., Xiujuan, D., Akif, K., Douglas, M.: A Surface-stress-based microcantilever aptasensor. IEEE Trans. Biomed. Circuits Syst. 8, 15-24 (2014)

5. Dareing, D.W., Thundat, T.: Simulation of adsorption-induced stress of a microcantilever sensor. J. Appl. Phys. 97, 043526 (2005)

6. Zhang, J.Q., Yu, S.W., Feng, X.Q., Wang, G.F.: Theoretical analysis of adsorption-induced microcantilever bending. J. Appl. Phys. 103, 093506 (2008)

7. Sa, C.P.M., Varghese, B., Baby, A.: A review on functionally graded materials. Int. J. Eng. Sci. 3, 90-101 (2014)

8. Marur, P.R., Tippur, H.V.: Evaluation of Mechanical Properties of Functionally Graded Materials. J. Test. Eval. 26, 539-545 (1998)

9. Knoppers, G.E., Gunnink, J.W., Hout, J., Vliet, W.P.: The reality of functionally graded material products. In: International Solid Freeform Fabrication Symposium, Austin, Texas, August 2-4, pp. 38-43 (2004)

10. Rahimi, G.H., Davoudinik, A.R.: Large deflection of functionally graded cantilever flexible beam with geometric nonlinearity: analytical and numerical approaches. Sci. Iran. 17, 25-40 (2010)

11. Asghari, M., Ahmadian, M.T., Kahrobaiyan, M.H., Rahaeifard, M.: On the size-dependent behavior of functionally graded micro-beams. Mater. Des. 31, 2324-2329 (2010)

12. Asghari, M., Rahaeifard, M., Kahrobaiyan, M.H., Ahmadian, M.T.: The modified couple stress functionally graded Timoshenko beam formulation. Mater. Des. 32, 1435-1443 (2011)

13. Farokhi, H., Ghayesh, M.H., Gholipour, A.: Dynamics of functionally graded micro-cantilevers. Int. J. Eng. Sci. 115, 117-130 (2017) 
14. Rokni, H., Milani, A.S., Seethaler, R.J.: Size-dependent vibration behavior of functionally graded CNT-Reinforced polymer microcantilevers: Modeling and optimization. Eur. J. Mech. A/Solids 49, 26-34 (2015)

15. Rahi, A.: Vibration analysis of multiple-layer microbeams based on the modified couple stress theory: analytical approach. Arch. Appl. Mech. (2020). https://doi.org/10.1007/s00419-020-01795-z

16. Karhade, O.G., Chiluveru, S.S., Apte, P.R.: Novel cantilever for biosensing applications. In: 2004 IEEE/SEMI Advanced Semiconductor Manufacturing Conference and Workshop (IEEE Cat. No. 04CH37530). https://doi.org/10.1109/ASMC.20 04.1309606 (2004)

17. Vinson, J.R.: Plate and Panel Structures of Isotropic, Composite and Piezoelectric Materials, Including Sandwich Construction (Solid Mechanics and Its Applications), vol. 120. Springer, The Netherlands. ISBN 978-1-4020-3110-6 (2005)

18. Ansari, M.Z., Bisen, M., Bera, U.N., Kumar, S.: Simulation of surface stress-induced deflections in microcantilever biochemical sensor. J. Mech. Eng. Autom. 5, 23-26 (2015)

19. Goodno, B.J., Gere, J.M.: Mechanics of materials. Cengage Learning. SBN: 978-1-337-09334-7 (2018)

20. Shabana, Y.M., Noda, N.: Thermo-elasto-plastic stresses of functionally graded material plate with a substrate and a coating. J. Therm. Stresses 25, 1133-1146 (2002)

Publisher's Note Springer Nature remains neutral with regard to jurisdictional claims in published maps and institutional affiliations. 